Chem. Pharm. Bull.

35(10)4241-4248(1987) $]$

\title{
Conjugative Metabolism of 4-Methylumbelliferone in the Rat Liver: Verification of the Sequestration Process in Multiple Indicator Dilution Experiments
}

\author{
Seiji Miyauchi, ${ }^{*}$ Yuichi Sugiyama, Yasufumi Sawada, \\ Tatsuji IGa, and Manabu Hanano
}

Faculty of Pharmaceutical Sciences, University of Tokyo, Bunkyo-ku, Tokyo 113, Japan

(Received January 13, 1987)

\begin{abstract}
By means of the multiple indicator dilution (MID) method, the sequestration process of 4methylumbelliferone (4-MU), which is well known to be conjugated to glucuronide (4-MUG) and sulfate (4-MUS) in the rat liver, was found to be saturated as the dose was increased ( $J$. Pharmacokin. Biopharm., 15, 25 (1987). This observation might be attributed to the saturation of the conjugative metabolism. In the present study, using an in vivo tissue sampling single injection technique, we determined whether the sequestration process obtained by means of the MID method reflected the conjugative metabolism process. When the dose of 4-MU was increased from a low

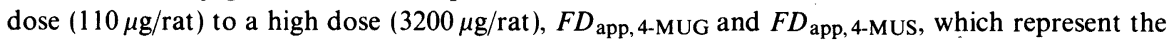
fractions of the amounts of the formed 4-MUG and 4-MUS remaining in the liver, respectively, remarkably decreased. Since 4-MUG and 4-MUS formed from 4-MU have low diffusional clearances between the blood and hepatocytes, they are effectively trapped in the hepatocytes and

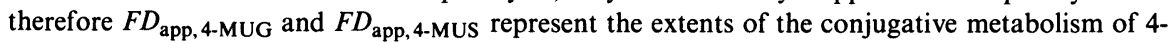
MU. Therefore, the dose-dependent change in the sequestration process was found to be attributable to the saturation of the conjugative metabolism. This observation confirms our previous hypothesis that the saturation of the sequestration process observed by using the MID method reflected that of the conjugative metabolism. Furthermore, according to the "sinusoidal" model, we simulated the time profiles of the amounts of drugs remaining in the liver, using parameters obtained from the MID data. The model, which has 20 sinusoidal compartments and can be regarded as identical to a "parallel tube" model, could reliably predict the time profiles of the amounts of drugs remaining in the liver.
\end{abstract}

Keywords - multiple indicator dilution method; sequestration; conjugative metabolism; sinusoidal model

\section{Introduction}

The rapid, single injection multiple indicator dilution (MID) method developed by Goresky et al. ${ }^{1-3)}$ is a very useful and elegant technique for the investigation of the uptake of materials from blood into the liver. It has provided an insight into the resistance to the influx and efflux processes at the cell membranes, in both the absence and presence of sequestration processes (intracellular metabolism, biliary excretion and/or intracellular transport). However, the parameters of the sequestration process obtained from the MID data in the case of low-clearance drugs, such as warfarin ${ }^{4)}$ and tolbutamide, ${ }^{5)}$ were usually much larger than those obtained from in vivo experiments, ${ }^{6,7)}$ since this method is performed within such a short period ( $30 \mathrm{~s})$ that neither metabolism nor biliary excretion can be entirely determined for such low-clearance drugs. Unfortunately, it is still not clear what sequestration process is being measured, but in the case of a high-clearance drug, the sequestration process observed by means of the MID method might be considered to reflect metabolism and/or excretion processes. The purpose of the present study was to determine whether the sequestration 
process obtained by using the MID method does reflect these processes in the case of a highclearance drug.

4-Methylumbelliferone (4-MU), which is used therapeutically as a cholagogue, is well known to be substantially cleared by conjugative metabolism in the liver. It has often been used as a model compound in conjugation studies. ${ }^{8,9)}$ In our previous study, we found a remarkable dose-dependency in the hepatic elimination of 4-MU. ${ }^{10)}$ Moreover, utilizing the MID method, we found that the limiting step of the apparent hepatic intrinsic clearance of 4MU changed with increasing dose owing to the saturation of the sequestration process. ${ }^{11}$ ) Although we considered that the saturation of the sequestration process might be attributed to that of the conjugative metabolism, we have not demonstrated yet whether the metabolism of 4-MU actually occurs in such a short period (30 s). In the present study, using an in vivo tissue sampling single injection technique developed by Pardridge et al. ${ }^{12-15}$ ) we examined whether conjugative metabolism occurred within such a short period. Furthermore, using the parameters obtained from the MID data, we simulated the time profiles of the amounts of drugs remaining in the liver, according to the "sinusoidal" model, ${ }^{16)}$ which breaks up the sinusoid into a large number of sequentially perfused compartments with accompanying cellular compartments.

\section{Experimental}

Materials - 4-MU, its glucuronide (4-MUG) and sulfate (4-MUS) and bovine serum albumin (BSA) (fraction V) were purchased from Sigma Chemical Co. (St. Louis, MO). Beta-Glucuronidase/arylsulfatase was purchased from Boehringer Mannheim GmbH (Mannheim, West Germany). All other reagents were commercial products of analytical grade.

In Vivo Tissue-Sampling Single Injection Technique-The technique used in the present study was basically according to Pardridge et al. ${ }^{12-15)}$ Male Wistar rats (weighing $250-300 \mathrm{~g}$ ) maintained on a normal laboratory diet, were used throughout. Under light ether anesthesia, a midline abdominal incision was made and the hepatic artery was ligated. Immediately after ligation of the hepatic artery, the portal vein was canulated with a 25-gauge needle and $300 \mu \mathrm{l}$ of the injection solution (low dose, $110 \mu / \mathrm{rat}$; high dose, $3200 \mu \mathrm{g} / \mathrm{rat}$ ) was rapidly injected. The injection solution was made by mixing equal volumes of rat plasma and Krebs-Ringer buffer containing the test substance. At particular times $(20,40$ and $60 \mathrm{~s})$ after portal injection, the portal vein was rapidly cut off and the whole liver tissue was excised. Immediately after excision, the whole tissue was frozen in a bath of acetone cooled to $-70^{\circ} \mathrm{C}$ with dry ice and kept at $-40^{\circ} \mathrm{C}$ until assayed. The amounts of 4-MU taken up into the liver and its metabolites generated intracellularly were determined individually by using thin layer chromatography as described in detail previously. ${ }^{17}$

The fractions of the dose for 4-MU, 4-MUG and 4-MUS remaining in the liver after injection of 4-MU are designated as $F D_{\text {app,4-MU }}, F D_{\text {app,4-Mug }}$ and $F D_{\text {app,4-mus }}$, respectively. It is assumed that no biliary excretion of the parent drug (4-MU) or its metabolites (4-MUG and 4-MUS) occurs during such a short period. No metabolism other than sulfation and glucuronidation occurs in the body. ${ }^{10)}$ Since 4-MUG and 4-MUS formed from 4-MU may be effectively trapped in the liver due to their low diffusional clearances between the blood and hepatocytes, ${ }^{17}$ $F D_{\text {app,4-Mug }}$ and $F D_{\text {app,4-mus }}$ may reflect the extents of the conjugative metabolism of 4-MU. Therefore, the sum of

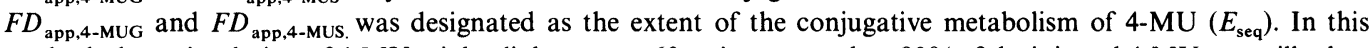
method, the recirculation of 4-MU might slight even at $60 \mathrm{~s}$, since more than $90 \%$ of the injected 4-MU was still taken up in the liver at $40 \mathrm{~s}$ and the steady-state distribution volume of $4-\mathrm{MU}(290 \mathrm{ml} / \mathrm{kg})$ was very large. $\left.{ }^{10}\right)$

Furthermore, according to the "sinusoidal" model, ${ }^{16)}$ we simulated the time profiles of the amounts of drugs remaining in the liver, using parameters obtained from the MID data. The "sinusoidal" model breaks up the sinusoid into a large number of sequentially perfused compartments with accompanying cellular compartments. The system used for simulation was a portal vein bolus injection. For the sake of simplicity, several assumptions were made, as follows; no biliary excretion is assumed for the parent drug (4-MU) and its metabolites (4-MUG and 4-MUS) during such a short period. The volumes of blood and the liver, and the intrinsic clearance for the influx, efflux and sequestration processes are identical with regard to all compartments.

In the case of the $n$-compartment sinusoidal model, in which the sinusoid is broken up into $n$ sequentially perfused compartments with accompanying cellular compartments, the following mass-balance equations hold in the $i$-th compartment: for the extracellular space

$$
\left(V_{\mathrm{E}} / n\right) \frac{d C_{\mathrm{B}, i}}{d t}=-\left(Q+f_{\mathrm{B}} \cdot C L_{\mathrm{in}, n}^{\mathrm{inf}}\right) \cdot C_{\mathrm{B}, i}+C L_{\mathrm{int}, n}^{\mathrm{eff}} \cdot C_{\mathrm{L}, i}+Q \cdot C_{\mathrm{B}, i-1}
$$


and for the intracellular space

$$
\left(V_{\mathrm{L}} / n\right) \frac{d C_{\mathrm{L}, i}}{d t}=f_{\mathrm{B}} \cdot C L_{\mathrm{int}, n}^{\mathrm{inf}} \cdot C_{\mathrm{B}, i}-\left(C L_{\mathrm{int}, n}^{\mathrm{eff}}+C L_{\mathrm{int}, n}^{\mathrm{seq}}\right) \cdot C_{\mathrm{L}, i}
$$

where $Q$ is the hepatic blood flow; $t$ is the time; $C_{\mathrm{B} i}$ and $C_{\mathrm{L} i}$ are the concentrations in the extracellular and intracellular spaces of the $i$-th compartment $(i=1 \rightarrow n)$, respectively; $f_{\mathrm{B}}$ represents the unbound fraction in the blood $\left(f_{\mathrm{B}}=0.91\right.$; in vivo experiments $\left.{ }^{17)}\right)$ or the perfusate $\left(f_{\mathrm{B}}=0.88\right.$; perfusion experiments $\left.{ }^{11)}\right) ; V_{\mathrm{E}}$ and $V_{\mathrm{L}}$ are the volumes of the extracellular and intracellular spaces, respectively; $C L_{\mathrm{int}, n}^{\mathrm{inf}}, C L_{\mathrm{int}, n}^{\text {eff }}$ and $C L_{\mathrm{in} t, n}^{\mathrm{seq}}$ are the intrinsic clearances for the influx, efflux and sequestration processes in the $n$-compartment sinusoidal model, respectively. In the case of a portal vein injection, the above equations are integrated from $t=0$ to $\infty$. Subsequently, rearranging all equations, we obtained the dose-normalized $A U C_{n}$ (area under the outflow fraction of dose (per ml) v.s. time) of the $n$-th compartment as follows:

$$
\begin{aligned}
& A U C_{n}=\frac{Q^{n}}{\left(Q+f_{\mathrm{B}} \cdot C L_{\mathrm{int}, n}^{\mathrm{app}}\right)^{n}} \\
& C L_{\mathrm{int}, n}^{\mathrm{app}}=\frac{C L_{\mathrm{int}, n}^{\mathrm{inf}} \cdot C L_{\mathrm{int}, n}^{\mathrm{seq}}}{C L_{\mathrm{int}, n}^{\mathrm{eff}}+C L_{\mathrm{int}, n}^{\mathrm{seq}}}
\end{aligned}
$$

where $C L_{\mathrm{int}, n}^{\mathrm{app}}$ represents the apparent intrinsic clearance in the $n$-compartment sinusoidal model. Since $A U C_{n}$ is identical to the instantaneous hepatic availability $(F)$, the instantaneous hepatic extraction ratio $(E)$ is expressed as follows:

$$
E=1-\frac{Q^{n}}{\left(Q+f_{\mathrm{B}} \cdot C L_{\mathrm{int}, n}^{\mathrm{app}}\right)^{n}}
$$

Rearranging Eq. 5 to solve for $C L_{\mathrm{int}, n}^{\mathrm{app}}$ yields:

$$
f_{\mathrm{B}} \cdot C L_{\mathrm{int}, n}^{\mathrm{app}}=\frac{1-\sqrt[n]{1-E}}{Q \sqrt[n]{1-E}}
$$

Using the previously reported $E$ values $\left(E=0.89\right.$ and 0.28 at low and high doses, respectively), ${ }^{11)}$ the values of $f_{\mathrm{B}} \cdot C L_{\mathrm{int}, n}^{\mathrm{app}}$ were calculated at the low and high doses according to Eq.6. Although in our previous experiment, ${ }^{11)}$ the low dose was $50 \mu \mathrm{g} / \mathrm{rat}$, we chose $110 \mu \mathrm{g} / \mathrm{rat}$ as the low dose in the present study, taking account of the sensitivity of the determination method of drugs in the liver. According to our preliminary experiments (unpublished observations), the extraction ratio of $4-\mathrm{MU}$ was almost constant when the dose was increased to $300 \mu \mathrm{g} / \mathrm{rat}$. Therefore, in the present case $(110 \mu \mathrm{g} / \mathrm{rat})$, kinetic parameters obtained from the MID analysis at the dose of $50 \mu \mathrm{g}$ could be used for the simulation. Subsequently, assuming that the ratio among $f_{\mathrm{B}} \cdot C L_{\mathrm{int}, n}^{\mathrm{inf}}: C L_{\mathrm{int}, n}^{\mathrm{eff}}: C L_{\mathrm{int}, n}^{\mathrm{seq}}$ is identical to that among the influx : efflux : sequestration intrinsic clearances $\left(C L_{\text {int,inf }}, C L_{\text {int,eff }}\right.$ and $C L_{\text {int,seq }}$, respectively) obtained by means of the MID method, we obtained the parameters $\left(f_{\mathrm{B}} \cdot C L_{\mathrm{int}, n}^{\mathrm{inf}}, C L_{\mathrm{int}, n}^{\text {eff }}\right.$ and $\left.C L_{\mathrm{int}, n}^{\text {seq }}\right)$, using the following ratios ${ }^{11)}$ :

$$
\begin{aligned}
& \text { in the case of the low dose: } f_{\mathrm{B}} \cdot C L_{\mathrm{int}, n}^{\mathrm{inf}}: C L_{\mathrm{int}, n}^{\mathrm{eff}}: C L_{\mathrm{int}, n}^{\mathrm{seq}}=4.7: 1: 1 \\
& \text { in the case of the high dose: } f_{\mathrm{B}} C L_{\mathrm{int}, n}^{\mathrm{inf}}: C L_{\mathrm{int}, n}^{\mathrm{eff}}: C L_{\mathrm{int}, n}^{\mathrm{seq}}=15: 5.0: 1
\end{aligned}
$$

The fraction of the dose for 4-MU $\left(F D_{\text {app,4-MU }}\right)$ and the extent of the conjugative metabolism $\left(E_{\text {seq }}\right)$ were obtained by numerically solving all differential equations as described above from $t=0$ to $60 \mathrm{~s}$, by the Runge-Kutta-Gill method at a suitable interval (1/1200 of $60 \mathrm{~s})$ using a microcomputer. Parameters used in this simulation are as follows:

(1) $f_{\mathrm{B}} \cdot C L_{\mathrm{int}, n}^{\mathrm{inf}}, C L_{\mathrm{int}, n}^{\mathrm{eff}}$ and $C \dot{L}_{\mathrm{int}, n}^{\text {seq }}$ were calculated as described above.

(2) $Q$ is $0.025 \mathrm{ml} / \mathrm{s} \mathrm{g} \mathrm{liver.}{ }^{18,19)}$

(3) $V_{\mathrm{E}}$ and $V_{\mathrm{L}}$ are $0.227 \mathrm{ml} / \mathrm{g}$ liver and $0.593 \mathrm{ml} / \mathrm{g}$ liver, respectively. ${ }^{11)}$

\section{Results}

\section{In Vivo Tissue Sampling Single Injection Technique}

The time courses of the fractions of the amount of 4-MU ( $\left.F D_{\text {app,4-MU }}\right)$ and the sum of those of its formed metabolite (4-MUG and 4-MUS) remaining in the liver $\left(E_{\text {seq }}\right)$ at 20, 40 and $60 \mathrm{~s}$ after the portal vein injection are summarized in Table I. The value of $E_{\text {seq }}$ decreased remarkably as the dose was increased. Previously, we could not detect the conjugative 
TABLE I. Results of the Uptake Study by an in Vivo Tissue-Sampling Single Injection Technique

\begin{tabular}{|c|c|c|c|c|c|}
\hline Dose & Time & $F D_{\text {app }, 4-\mathrm{MU}^{a)}}$ & $F D_{\text {app,4-MUG }}^{a)}$ & $F D_{\text {app,4-MUs }}^{a)}$ & $E_{\text {seq }}^{b)}$ \\
\hline Low & 20 & $0.250 \pm 0.021$ & $0.507 \pm 0.135$ & $0.183 \pm 0.001$ & $0.690 \pm 0.100$ \\
\hline \multirow{2}{*}{$(110)$} & 40 & $0.195 \pm 0.019$ & $0.419 \pm 0.088$ & $0.224 \pm 0.014$ & $0.643 \pm 0.066$ \\
\hline & 60 & $0.078 \pm 0.021$ & $0.484 \pm 0.086$ & $0.187 \pm 0.051$ & $0.671 \pm 0.112$ \\
\hline High & 20 & $0.841^{\circ} \pm 0.088^{c)}$ & $0.062 \pm 0.005^{c)}$ & $0.018 \pm 0.002^{c)}$ & $0.080 \pm 0.005^{c)}$ \\
\hline \multirow[t]{2}{*}{$(3200)$} & 40 & $0.705 \pm 0.056^{c)}$ & $0.095 \pm 0.004^{c)}$ & $0.017 \pm 0.002^{c)}$ & $0.109 \pm 0.002^{c)}$ \\
\hline & 60 & $0.448 \pm 0.135^{c)}$ & $0.124 \pm 0.009^{c)}$ & $0.018 \pm 0.002^{c)}$ & $0.142 \pm 0.006^{c}$ \\
\hline
\end{tabular}

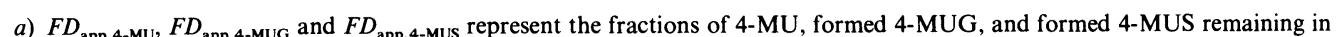
the liver, respectively. The parameters were expressed as mean \pm S.E. $(n=3) . \quad b) E_{\text {seq }}$ was designated as the sum of $F D_{\text {app, } 4-\mathrm{Mug}}$ and

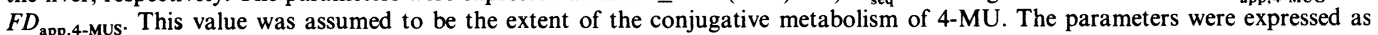
mean \pm S.E. $(n=3)$. c) Statistically significant $(p<0.01)$ difference from the data at low dose.

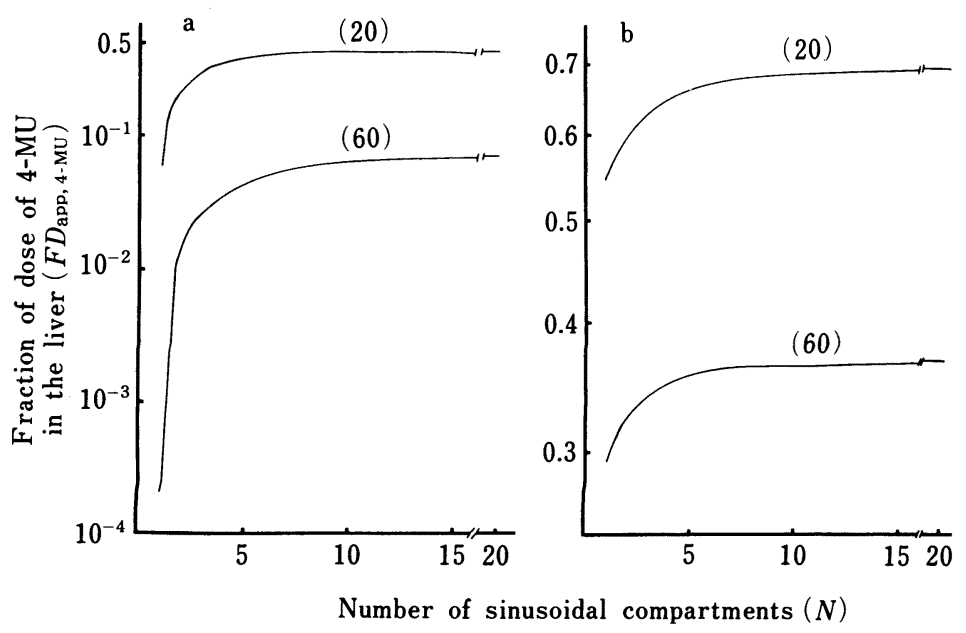

Fig. 1. Relationship between the Fraction of the Amounts of 4-MU in the Liver ( $F D_{\text {app,4-mU }}$ ) and the Number of the Sinusoidal Compartments $(N)$

Panel a, low dose (110 $\mu \mathrm{g})$; panel b, high dose $(3200 \mu \mathrm{g})$. The numbers on figures show the time (s) after the portal vein bolus injection.

metabolites in the effluent perfusate within a short period $(<30 \mathrm{~s})$ by means of the MID method. ${ }^{11)}$ 4-MUG and 4-MUS were found to show low diffusional clearance between the blood and hepatocytes by using isolated hepatocytes. Consequently, the fractions of the amounts of 4-MUG and 4-MUS remaining in the liver $\left(F D_{\text {app,4-MUG }}, F D_{\text {app,4-MUS }}\right.$, respectively) were considered to reflect the extents of the conjugative metabolism, on the ground that 4MUG and 4-MUS formed from 4-MU were effectively trapped in the hepatocytes by the diffusional barrier between the blood and hepatocytes. ${ }^{17}$ ) Thus, it was suggested that saturation of the conjugative metabolism actually occurred for a short period when the dose was increased, and the saturation of the sequestration process observed by means of the MID method might reflect that of the conjugative metabolism. The values of $F D_{\text {app,4-MUG }}$ at the low dose and $F D_{\text {app,4-mus }}$ at the low and high doses were almost constant irrespective of time, whereas the value of $F D_{\text {app,4-MU }}$ decreased with time at both doses. On the other hand, the values of $F D_{\text {app,4-Mug }}$ at the high dose gradually increased with time, whereas the value of $F D_{\text {app,4-MU }}$ decreased. 


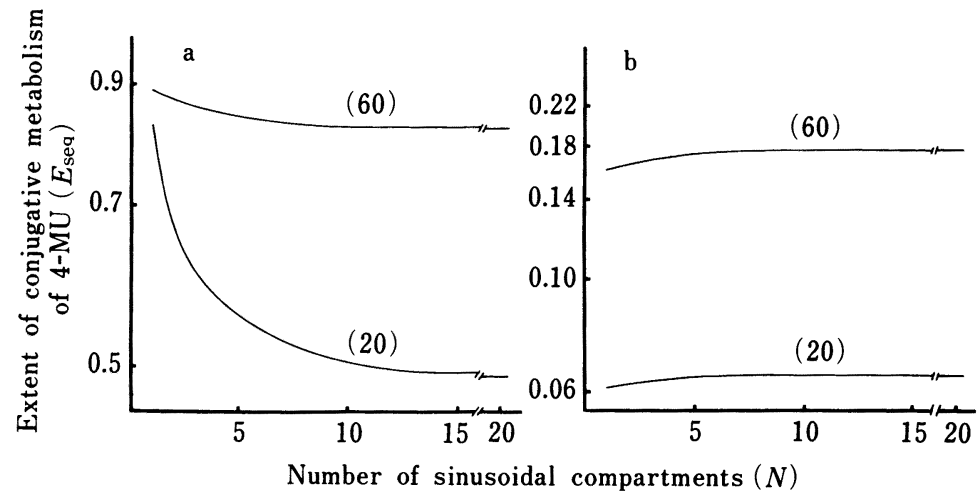

Fig. 2. Relationship between the Extent of Conjugative Metabolism $\left(E_{\mathrm{seq}}\right)$ and the Number of the Sinusoidal Compartments $(N)$

Panel a, low dose $(110 \mu \mathrm{g})$; panel $\mathrm{b}$, high dose $(3200 \mu \mathrm{g})$. The numbers on figures show the time (s) after the portal vein bolus injection.

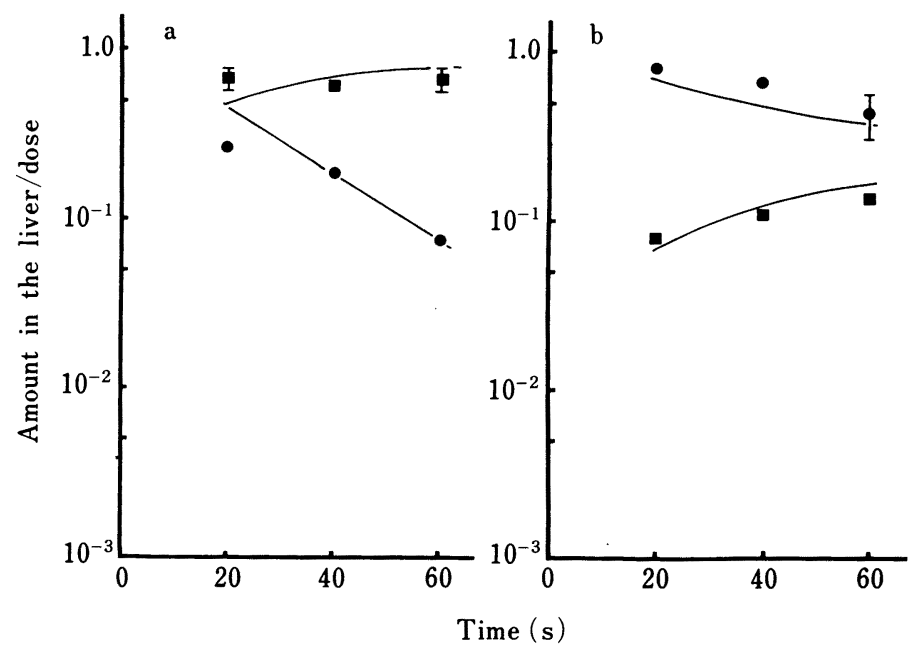

Fig. 3. Prediction of the 4-MU, Formed 4-MUG and Formed 4-MUS Remaining in the Liver Using the Parameters Obtained from the MID Data

Panel a, low dose injection $(110 \mu \mathrm{g})$; panel b, high dose injection $(3200 \mu \mathrm{g})$. The lines represent the simulation curves of the model, in which the sinusoid has 20 sinusoidal compartments. Each point represents the mean \pm S.E. of three experiments. (), 4-MU; (ם), 4-MUG + 4-MUS.

\section{Simulation Study}

According to the "sinusoidal" model, we simulated $F D_{\text {app,4-MU }}$ and $E_{\text {seq }}$, using the parameters obtained from the MID data. ${ }^{11)}$ The relationships between $F D_{\text {app,4-MU }}$ and $E_{\text {seq }}(20$ and $60 \mathrm{~s}$ after bolus portal vein injection) and the sinusoidal compartment number $(N)$ are depicted in Figs. 1 and 2. When the $N$ value became more than 7, the values of $F D_{\text {app,4-mu }}$ and $E_{\text {seq }}$ were almost constant irrespective of the $N$ value. Since the concentration gradient along the hepatic blood flow path in the "sinusoidal" model becomes sharper as the $N$ value is increased, this model might be considered to be identical with the "parallel" tube model, in the range of larger $N$ value (more than 7). The results of simulation with the model, in which the sinusoid has 20 sinusoidal compartments, at low and high doses are depicted in Fig. 3. This model could predict the values of $F D_{\text {app,4-MU }}$ and $E_{\text {seq }}$ well at both low and high doses. 


\section{Discussion}

The MID method has been extensively used to clarify the physiology of various organs such as the heart ${ }^{20)}$ kidney, ${ }^{21)}$ and liver. ${ }^{1-3)}$ This method has the advantage that three parameters with regard to the influx, efflux and sequestration (metabolism, biliary excretion and/or intracellular transport) processes are determined simultaneously and individually. However, the experimental period is so short that we cannot easily determine what the sequestration process is.

We will consider the relationship between the intrinsic clearance for the sequestration process observed by means of the MID method and that in vivo. The hepatic clearance $\left(C L_{\mathrm{H}}\right)$ is a function of the hepatic blood flow $\left(Q_{\mathrm{H}}\right)$, the blood unbound fraction $\left(f_{\mathrm{B}}\right)$ and the apparent intrinsic clearance $\left(C L_{\mathrm{int}, \mathrm{H}}^{\mathrm{app}}\right)$, and can be expressed according to the following two models ${ }^{22}$; namely, the "well-stirred" model, which describes the liver as a well-stirred compartment, and the "parallel tube" model, which describes the liver as a series of identical and parallel tubes with enzymes distributed evenly within hepatocytes lining the tubes. In these two models, $C L_{\mathrm{H}}$ is expressed by the following equations. ${ }^{23)}$ For the well-stirred model:

$$
C L_{\mathrm{H}}=\frac{Q_{\mathrm{H}} \cdot f_{\mathrm{B}} \cdot C L_{\mathrm{int}, \mathrm{H}}^{\mathrm{app}}}{Q_{\mathrm{H}}+f_{\mathrm{B}} \cdot C L_{\mathrm{int}, \mathrm{H}}^{\mathrm{app}}}
$$

For the parallel tube model:

$$
C L_{\mathrm{H}}=Q_{\mathrm{H}}\left(1-\exp \left(-\frac{f_{\mathrm{B}} \cdot C L_{\mathrm{int}, \mathrm{H}}^{\mathrm{app}}}{Q_{\mathrm{H}}}\right)\right)
$$

For either model, $C L_{\mathrm{int}, \mathrm{H}}^{\mathrm{app}}$ is expressed by

$$
C L_{\mathrm{int}, \mathrm{H}}^{\mathrm{app}}=\frac{C L_{\mathrm{int}}^{\mathrm{inf}} \cdot C L_{\mathrm{int}}^{\mathrm{seq}}}{C L_{\mathrm{int}}^{\mathrm{eff}}+C L_{\mathrm{int}}^{\mathrm{seq}}}
$$

where $C L_{\mathrm{int}}^{\mathrm{inf}}, C L_{\mathrm{int}}^{\mathrm{eff}}$ and $C L_{\mathrm{int}}^{\mathrm{seq}}$ represent the intrinsic clearances for the influx, efflux and sequestration processes, respectively.

Using the reported values obtained from an in vivo experiment, ${ }^{6)}$ we estimated the value of $f_{\mathrm{B}} \cdot C L_{\mathrm{int}, \mathrm{H}}^{\mathrm{app}}$ for a low-clearance drug, warfarin, which is metabolized only by the liver and is used therapeutically as an anticoagulant drug. The total body clearance $\left(C L_{\text {tot }}\right)$ calculated from $A U C_{\text {iv }}$ (area under plasma concentration $v s$. time curve after intravenous injection) was very small $(0.0025-0.010 \mathrm{ml} / \mathrm{min})$ relative to $Q_{\mathrm{H}}(12-16 \mathrm{ml} / \mathrm{min})$. The values of $f_{\mathbf{B}} \cdot C L_{\mathrm{int}, \mathrm{H}}^{\mathrm{app}}$ for such a low-clearance drug (warfarin) in both models are identical to that of $C L_{\mathrm{tot}}$. On the other hand, the value of $f_{\mathrm{B}} \cdot C L_{\mathrm{int}, \mathrm{H}}^{\mathrm{app}}$ for warfarin calculated using parameters obtained from the MID data ${ }^{7)}$ according to Eq. 9 , was much larger $(10.1-25.4 \mathrm{ml} / \mathrm{min})$ than that obtained from in vivo experiment. This overestimation might be related to the uncertainty in the kinetic parameter for the sequestration process during such a short experiment period $(<30 \mathrm{~s})$, since warfarin is a slowly metabolized drug. We found a similar overestimation in $f_{\mathrm{B}} \cdot C L_{\mathrm{int}, \mathrm{H}}^{\mathrm{app}}$ obtained from the MID method in the case of tolbutamide. ${ }^{5,7)}$

Recently, we used the MID method to investigate the dose-dependency of the hepatic elimination for 4-MU, which is well known to be conjugated to glucuronide (4-MUG) and sulfate (4-MUS). In this experiment, we found that the sequestration process was saturated as the dose was increased, suggesting that the saturation of the sequestration process might be due to that of the conjugative metabolism. However, we could not measure the conjugative metabolites (4-MUG and 4-MUS) in the effluent perfusate and in the liver within such a short period $(<30 \mathrm{~s})$ and therefore, strictly speaking, we could not determine what the sequestration process reflected. In the present study, we examined whether conjugative metabolism occurred in the liver within such a short period by employing an in vivo tissue-sampling single injection 
technique. As shown in Table I, the value of $E_{\text {seq }}$ at the low dose (0.69) was much higher than that at the high dose $(0.08)$ at $20 \mathrm{~s}$. Thus, it was suggested that conjugative metabolism actually occurred during this short period and was saturated at the high dose. At the low dose, the value of $E_{\mathrm{seq}}$ was constant independent of time, whereas the value of $F D_{\mathrm{app}, 4-\mathrm{MU}}$ decreased with time (Table I). In contrast, at the high dose, $E_{\text {seq }}$ gradually increased, whereas the value of $F D_{\text {app,4-MU }}$ decreased as in the case of the low dose, although the absolute values of $F D_{\text {app,4-Mu }}$ were different between the low and high doses (Table I). The difference in $E_{\text {seq }}$ between the low and high doses might be explained as follows. At the low dose, 4-MU was removed to a large extent by the conjugative metabolism. On the other hand, at the high dose, a large amount of 4-MU remained in the liver due to the saturation of the conjugative metabolism. At the high dose, the conjugative metabolism might continue for $60 \mathrm{~s}$ and therefore, the value of $E_{\text {seq }}$ gradually increased with time. In contrast, the value of $F D_{\text {app,4-MU }}$ decreased with time due to both wash-out with the hepatic blood flow and conjugative metabolism.

Furthermore, according to the "sinusoidal" model, ${ }^{16)}$ we simulated the fraction of 4-MU remaining in the liver and the extent of its conjugative metabolism in the liver, using the parameters obtained from the MID data. The "sinusoidal" model breaks up the sinusoid into a large number of sequentially perfused compartments with accompanying cellular compartments. The number of perfused compartments $(N)$ within the sinusoid is an index of the degree of axial mixing in the model. Thus, as the $N$ value increases to infinity, this model may approach the "parallel tube" model. When the $N$ value was more than 7 , the value of $F D_{\text {app,4-Mu }}$ was constant irrespective of the $N$ value (Fig. 1). The value of $E_{\text {seq }}$ was also constant irrespective of the $N$ value, when the $N$ value was more than 7 (Fig. 2). Therefore, the sinusoidal model with more than 7 perfused compartments mimicks the "parallel tube" model. As shown in Fig. 3, the simulation curves based on the "sinusoidal" model with 20 perfused compartments predicted well the time-courses of the values of $F D_{\text {app,4-MU }}$ and $E_{\text {seq }}$ at both low and high doses. In the present study, the kinetic parameters obtained from the MID data were used to simulate the in vivo data according to a first order kinetic model. The best model in this simulation is a "flow-limited distributed" model, incorporating nonlinearity of the efflux and sequestration processes. However, such a calculation for the intracellular concentrations of drugs has not been successful so far, due to the mathematical difficulties. Therefore, a simpler "sinusoidal" model with first-order kinetics was used in the present study. A similar approach, in which the dose-dependent hepatic uptake of sulfobromophthalein, ${ }^{24)}$ indocyanine green ${ }^{25)}$ or bile acid $^{26)}$ was analyzed according to a first-order kinetic model, has often been used. Although such an approximation is convenient, we should be cautious in giving a quantitative interpretation to the kinetic parameters.

In conclusion, it is suggested that the sequestration rate constant obtained by means of the MID method for a highly cleared compound, 4-methylumbelliferone (4-MU), for a short period $(<30 \mathrm{~s})$ reflects the conjugative metabolic rate constant.

\section{References and Notes}

1) C. A. Goresky, W. H. Ziegler, and G. G. Bach, Circ. Res., 17, 739 (1970).

2) C. A. Goresky and B. E. Nadeau, J. Clin. Invest., 53, 634 (1973).

3) A. W. Wolkoff, C. A. Goresky, J. Sellin, Z. Gatmaitan, and I. M. Arias, Am. J. Physiol., 236, E638 (1979).

4) S. C. Tsao, Y. Sugiyama, Y. Sawada, S. Nagase, T. Iga, and M. Hanano, J. Pharmacokinet. Biopharm., 14, 51 (1986).

5) S. C. Tsao, Y. Sugiyama, Y. Sawada, T. Iga, and M. Hanano, Proceedings of the 104th Annual Meeting of Japanese Pharmaceutical Association, Sendai, 1984, p. 690.

6) A. Yacobi and G. Levy, J. Pharm. Sci., 66, 567 (1977).

7) O. Sugita, Y. Sawada, Y. Sugiyama, T. Iga, and M. Hanano, Drug Metab. Dispos., 12, 131 (1984). 
8) G. J. Mulder, S. Brouwer, J. G. Weitering, E. Scholtens, and K. S. Pang, Biochem. Pharmacol., 34, 1325 (1985).

9) B. Andersson, M. Berggren, and P. Moldeus, Drug Metab. Disposit., 6, 611 (1978).

10) K. Morita, Y. Sugiyama, and M. Hanano, J. Pharmacobio-Dyn., 9, 117 (1986).

11) S. Miyauchi, Y. Sugiyama, Y. Sawada, K. Morita, T. Iga, and M. Hanano, J. Pharmacokinet. Biopharm., 15, 25 (1987).

12) W. M. Pardridge and L. S. Jefferson, Am. J. Physiol., 228, 1155 (1975).

13) W. M. Pardridge, Am. J. Physiol., 232, E492 (1977).

14) W. M. Pardridge and L. J. Mietus, Am. J. Physiol., 237, E367 (1979).

15) W. M. Pardridge, A. J. Van Herle, R. T. Naruse, G. Flierer, and A. Costin, J. Biol. Chem., 258, 990 (1983).

16) R. A. Weisiger, C. M. Mendel, and R. R. Cavalieri, J. Pharm. Sci., 75, 233 (1986).

17) S. Miyauchi, Y. Sugiyama, Y. Sawada, T. Iga, and M. Hanano, J. Pharm. Sci., submitted.

18) C. V. Greenway and R. D. Stark, Physiol. Rev., 51, 23 (1971).

19) F. W. Ossenberg, P. Devis, and J. P. Benhamou, J. Appl. Physiol., 37, 806 (1974).

20) D. Cousineau, C. P. Rose, and C. A. Goresky, Circ. Res., 49, 501 (1981).

21) N. Ito, Y. Sawada, Y. Sugiyama, T. Iga, and M. Hanano, Am. J. Physiol., 251, F103 (1986).

22) K. S. Pang and M. Rowland, J. Pharmacokinet. Biopharm., 5, 625 (1977).

23) H. Sato, Y. Sugiyama, S. Miyauchi, Y. Sawada, T. Iga, and M. Hanano, J. Pharm. Sci., 75, 3 (1986).

24) C. A. Goresky, Am. J. Physiol., 207, 13 (1964).

25) G. Paumgartner and J. Reichen, Experientia, 31, 306 (1975).

26) J. Reichen and G. Paumgartner, Am. J. Physiol., 231, 734 (1976). 